\title{
Microgel-Stabilized Hydroxypropyl Methylcellulose and Dextran Water-in- Water Emulsion: Influence of pH, Ionic Strength and Temperature
}

Jinglin Zhang ${ }^{1}$, Lei Mei ${ }^{1}$, Peihua Ma ${ }^{1}$, Yuan Li ${ }^{2}$, Yang Yuan ${ }^{3}$, Qing-Zhu Zeng ${ }^{3}$, Qin Wang ${ }^{1 *}$

${ }^{1}$ Department of Nutrition \& Food Science, University of Maryland, College park, MD, 20742, U.S.

${ }^{2}$ College of Biosystems Engineering and Food Science, Zhejiang University, Hangzhou 310058, P.R. China

${ }^{3}$ School of Chemistry and Chemical Engineering, Guangzhou University, Guangzhou, 510006, P.R. China

*Corresponding author: Qin Wang, Associate Professor

Department of Nutrition \& Food Science

University of Maryland College Park

3106 Skinner Building

College Park, MD 20742

Phone: (301) 405-8421

E-mail: wangqin@umd.edu 


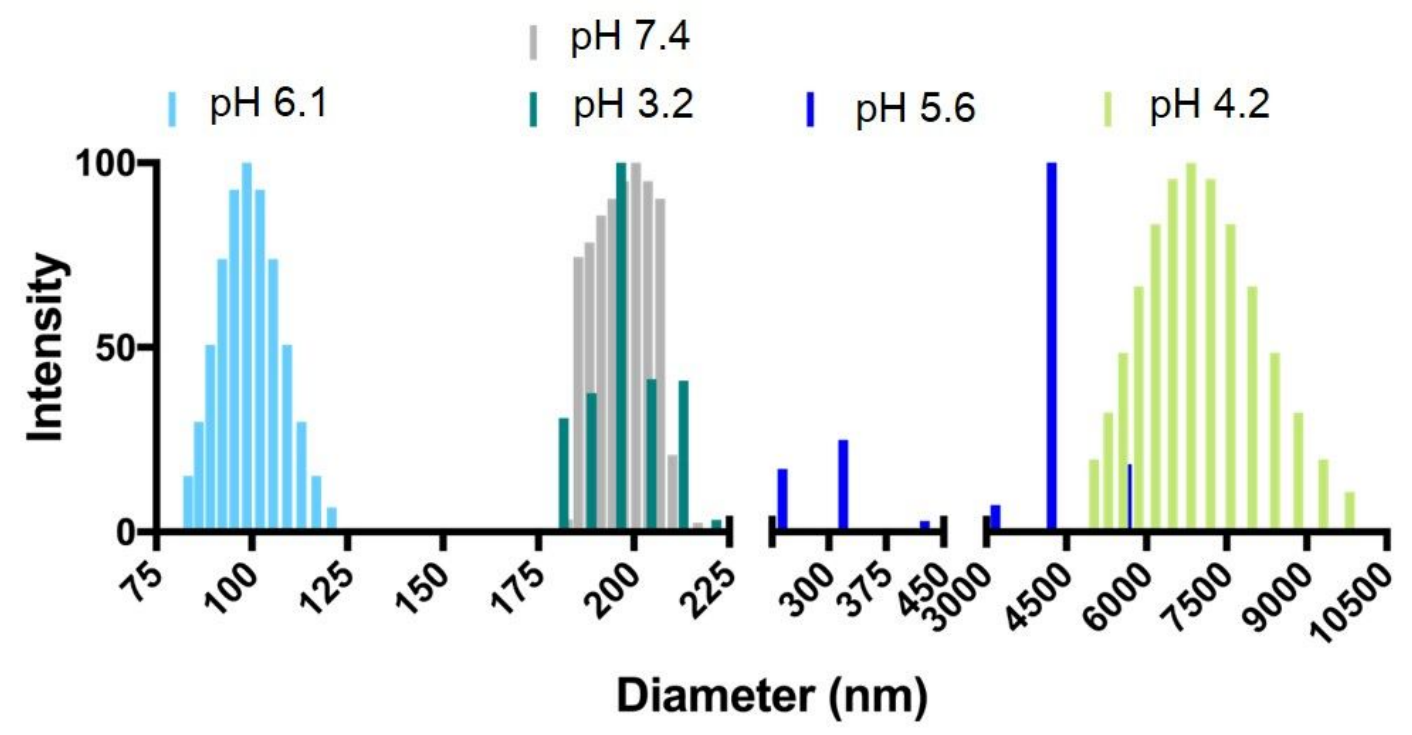

Figure S1. Particle size distribution of Blg microgels at various $\mathrm{pH}$ 


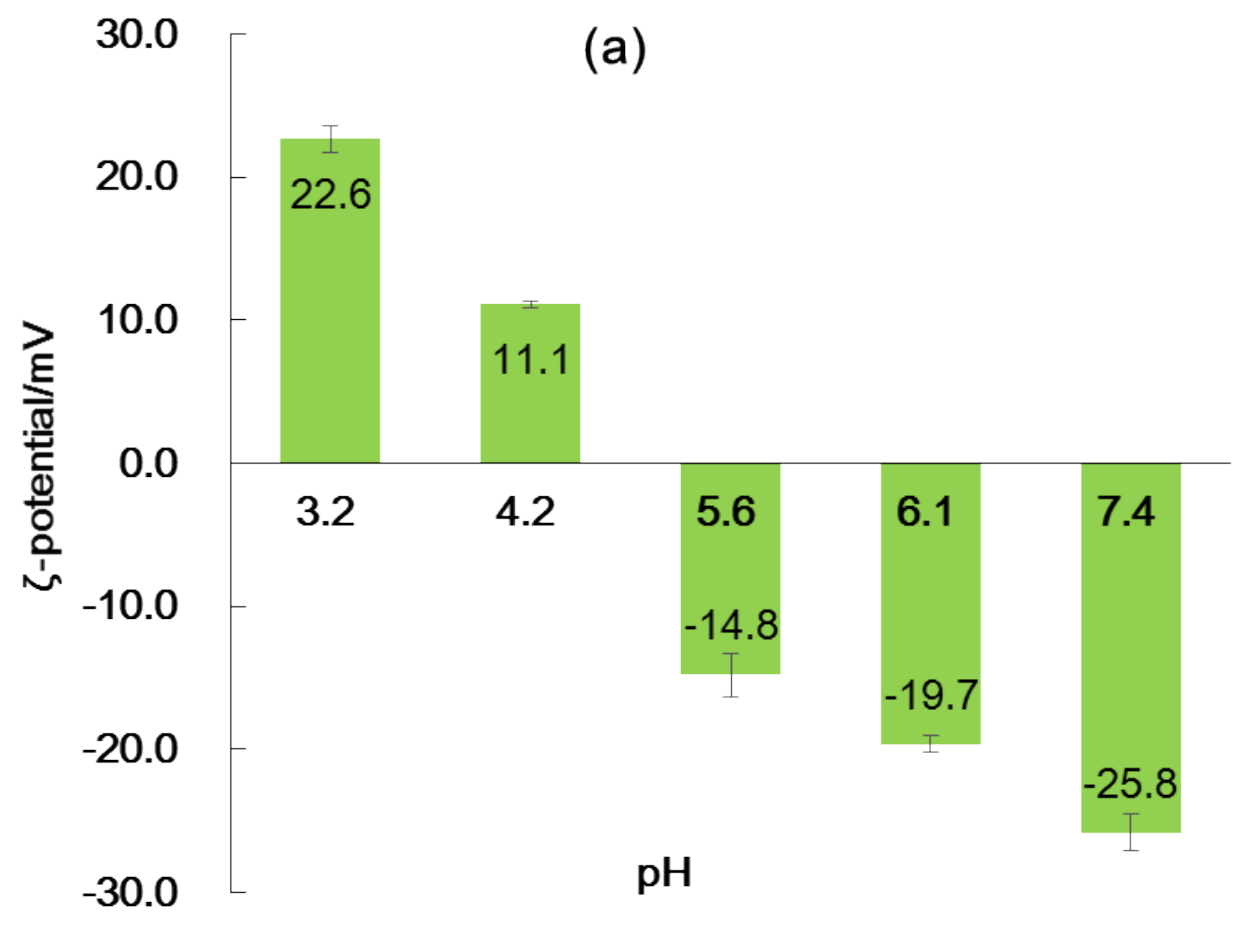

(b)

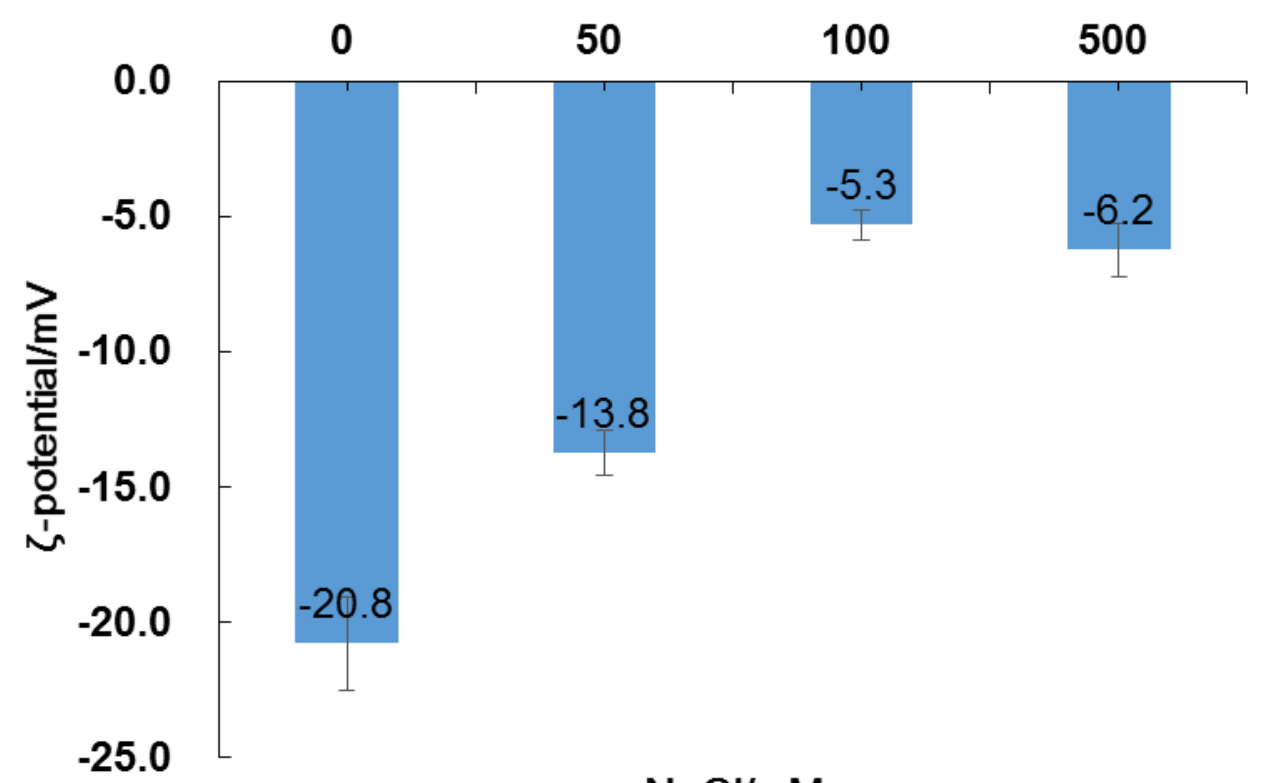

\section{$\mathrm{NaCl} / \mathrm{mM}$}

Figure S2. (a) Zeta-potential of Blg microgels at various $\mathrm{pH}$ and (b) ionic strength effect on zetapotential of Blg microgels in pH7.0 $20 \mathrm{mM} \mathrm{CPBuffer}$ 

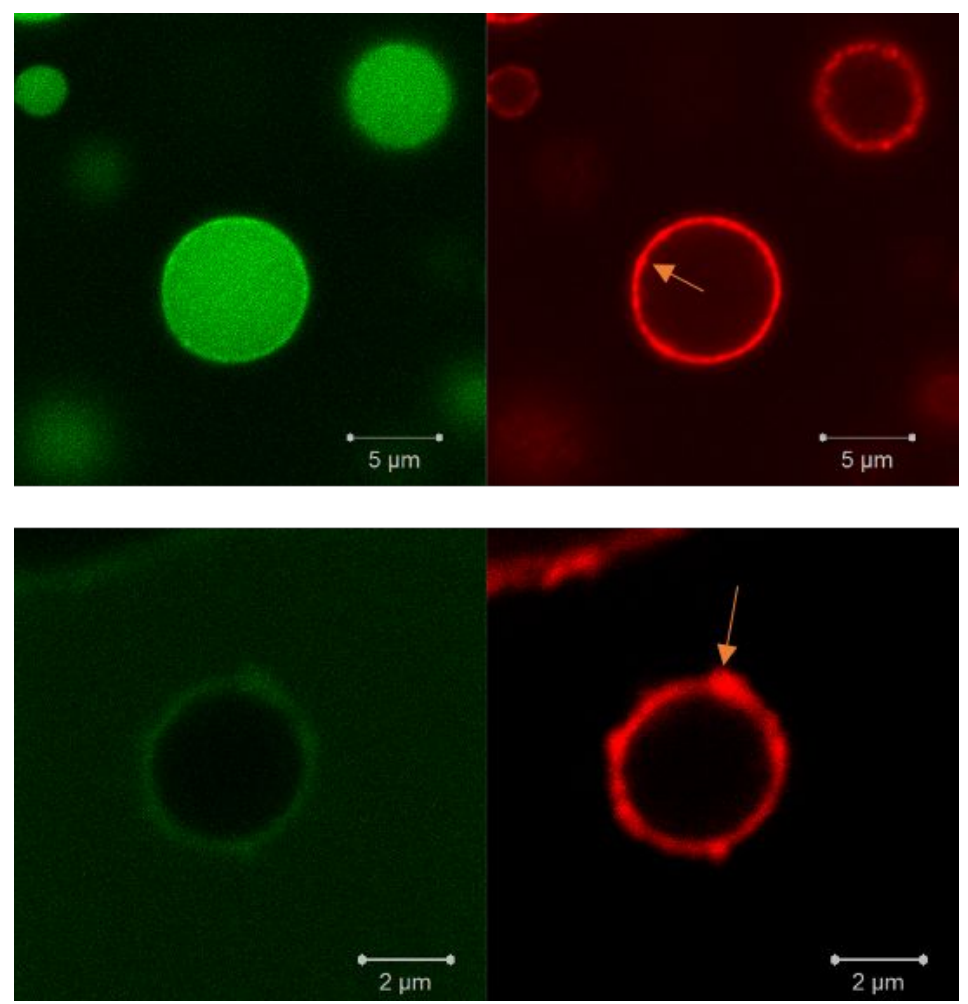

Figure S3. CLSM images of dextran in HPMC emulsion (top) and HPMC in dextran emulsion (bottom) in presence of protein microgels $(0.5 \%)$. Protein was dyed by rhodamine B (red) and dextran phase was FITC labelled (green). Yellow arrows indicated the microgels clusters. 


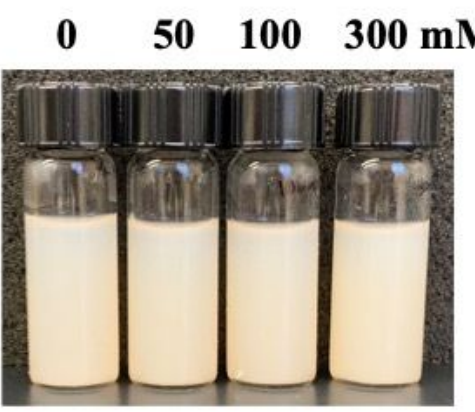

1 hour

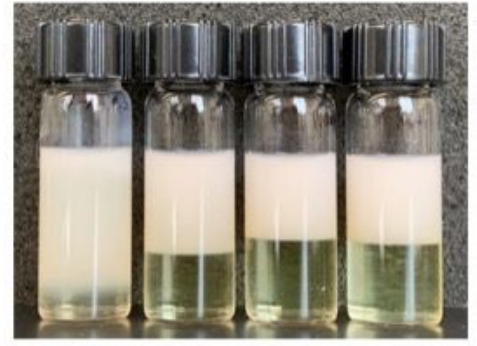

3 days

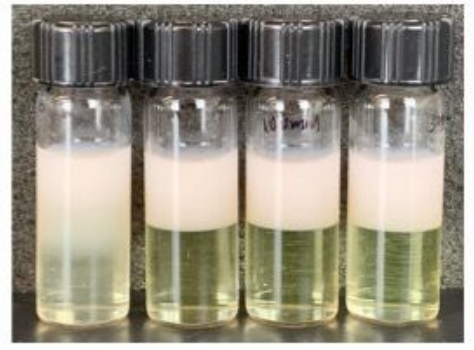

1 week

Figure S4. Evolution of HPMC/dextran (2\%/12\%) emulsions ( $\mathrm{pH} 3.0)$ at different ionic strength $(\mathrm{mM})$ containing $0.3 \%$ protein microgels with waiting time of 1 hour, 3 days and 1 week. 


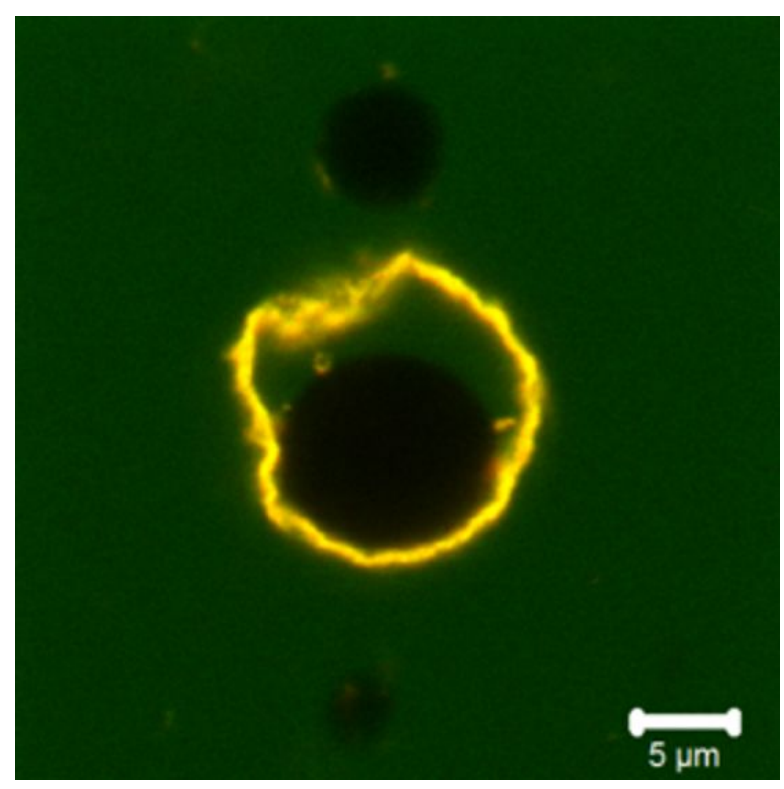

Figure S5. CLSM images of heated HPMC/dextran (1\%:12\%) emulsion with $0.25 \%$ protein microgels after $30 \mathrm{~min}$ thermal treatment at $90^{\circ} \mathrm{C}$ with addition of $2 \mathrm{mM}$ SDS solutions. The emulsion is colored by the presence of FITC-dextran (green) and rhodamine B dyed protein microgels (red). 
Table S1. Zeta-potential of dextran (Dex), HPMC and Blg microgels (BM) mixtures under different $\mathrm{pH}$ values.

\begin{tabular}{ccccc}
\hline \multirow{2}{*}{ Components } & \multicolumn{4}{c}{ Zeta Potential/mV } \\
\cline { 2 - 5 } & \multicolumn{2}{c}{$\mathrm{pH} \mathrm{3}$} & \multicolumn{2}{c}{$\mathrm{pH} \mathrm{7}$} \\
\cline { 2 - 5 } Avg. & Std. & Avg. & Std. \\
\hline \multirow{2}{*}{ Blg Microgels (BM) } & 21.37 & 2.10 & -27.21 & 0.80 \\
BM+Dextran & 20.40 & 0.14 & -28.60 & 0.43 \\
BM+HPMC & 20.03 & 0.29 & -27.13 & 0.83 \\
\hline
\end{tabular}

